\title{
How to Find More Supernovae with Less Work: Object Classification Techniques for Difference Imaging
}

\author{
S. Bailey ${ }^{1,4}$ C. Aragon, ${ }^{1}$ R. Romano, ${ }^{1,2}$ R. C. Thomas, ${ }^{1}$ B. A. Weaver ${ }^{1,3}$ D. Wong ${ }^{1}$
}

\begin{abstract}
We present the results of applying new object classification techniques to difference images in the context of the Nearby Supernova Factory supernova search. Most current supernova searches subtract reference images from new images, identify objects in these difference images, and apply simple threshold cuts on parameters such as statistical significance, shape, and motion to reject objects such as cosmic rays, asteroids, and subtraction artifacts. Although most static objects subtract cleanly, even a very low false positive detection rate can lead to hundreds of non-supernova candidates which must be vetted by human inspection before triggering additional followup. In comparison to simple threshold cuts, more sophisticated methods such as Boosted Decision Trees, Random Forests, and Support Vector Machines provide dramatically better object discrimination. At the Nearby Supernova Factory, we reduced the number of non-supernova candidates by a factor of 10 while increasing our supernova identification efficiency. Methods such as these will be crucial for maintaining a reasonable false positive rate in the automated transient alert pipelines of upcoming projects such as PanSTARRS and LSST.
\end{abstract}

Subject headings: methods: data analysis - methods: statistical — supernovae: general — techniques: image processing

\footnotetext{
${ }^{1}$ Lawrence Berkeley National Laboratory, 1 Cyclotron Road, Berkeley, CA 94720

${ }^{2}$ Luis W. Alvarez Fellow, National Energy Research Scientific Computing Center, 1 Cyclotron Road, Berkeley, CA 94720

${ }^{3}$ University of California, Space Sciences Laboratory, Berkeley, CA 94720

${ }^{4}$ Corresponding author: sjbailey@lbl.gov
} 


\section{Introduction}

Future large scale survey projects such as PanSTARRS1 and LSST2 2 are expected to generate automated rapid turnaround transient alerts for objects such as supernovae, active galactic nuclei, asteroids, Kuiper belt objects, and variable stars. They will do this by comparing new images to coadded stacks of reference images taken previously. Repeat observations of the same field will occur over timescales of minutes, hours, days, months, and years. Robust rejection of spurious non-astrophysical objects will be crucial to avoid excessive false positive alerts.

A major difficulty of current optical transient programs is the huge number of false positive objects which are difficult to reject while maintaining high selection efficiency for the real objects of interest. For example, the 2005 Sloan Digital Sky Survey II (SDSS-II) supernova program (Becker et al. 2006) required objects to be detected within 0.6 arcsec in at least two filters with signal-to-noise greater than 3 , yet this generated $\sim 4,000$ objects per night which needed to be visually checked by humans for verification. Their 2006 search drastically reduced this scanning load by requiring that all but the brightest objects be identified at the same location on multiple nights before they are passed to a human for verification. Although this reduced their scanning load, this method is not applicable to the real-time transient alert pipelines of PanSTARRS and LSST. A "60-second transient alert" would be meaningless if it really meant " $N$ days plus 60 seconds after the first positive identification." Although PanSTARRS and LSST will have multiple exposures of a field in the same night, this is equivalent to the multiple-filter requirement of the SDSS 2005 program which was still swamped by false positives.

This problem of false positives is not unique to nearby transient searches; it arises whenever a large number of objects are imaged, either from a wide-field survey or a deep narrow survey. The ESSENCE (Miknaitis et al. 2007) and SNLS (Astier et al. 2006) Canadian pipeline supernova searches both result in 100-200 objects to scan per night of data3. Although this is a manageable load for a current experiment, it would not scale to future surveys which will image thousands of square degrees per night 4 If current methods were used, the projects would need to drastically reduce their signal efficiency in order to maintain

\footnotetext{
${ }^{1}$ http://pan-starrs.ifa.hawaii.edu

${ }^{2}$ http://lsst.org

${ }^{3}$ private communication with W.M. Wood-Vasey (ESSENCE) and D. Balam (SNLS)

${ }^{4}$ For comparison, the SNLS supernova survey covers $\sim 1$ square degree per night, SDSS covers 150 square degrees per night, and SNfactory covers 350 to 850 square degrees per night.
} 
a manageable false-positive rate. The SNLS French pipeline uses multi-night data and an artificial neural net to select candidates for verification, but as noted above, using multi-night information is not applicable to rapid turnaround transient alert pipelines which intend to produce alerts within a minute of the first positive detection.

False positives arise from a variety of sources including diffraction spikes, saturated stars, optical ghosts, star halos, cosmic rays, satellite trails, CCD amplifier glow, other CCD artifacts, and image processing artifacts. In principle all of these effects are best identified and either fixed or masked at the image level. In practice there will always be effects which produce spurious detections. This problem is especially bad at the start of a search when covering new areas of sky, before consistent problems can be identified and masked. The goal of a classifier is to identify the real candidates of interest (signal events) while rejecting the spurious objects (background events).

In some cases, real astrophysical variable objects are the background events for other analyses. For example, asteroids, variable stars, and active galactic nuclei form a background for nearby supernova searches, yet they are the core science for other programs. This paper is written from the context of a nearby supernova search and thus these other astrophysical events are treated as background to reject, but the methods presented here are generally applicable to many object classification problems.

This paper presents the results of applying modern machine learning techniques to the

supernova search pipeline of the Nearby Supernova Factory (SNfactory, Aldering et al. 2002). $\$ 2$ presents a variety of machine learning techniques. \$3 describes the Nearby Supernova Factory search, and $\S \S 4$ and 5 present the training data and classification software used. $\$ 6$ compares the various methods. We find that methods such as Boosted Trees, Random Forests, and Support Vector Machines perform dramatically better than the threshold cuts which are typically used by supernova search programs.

\section{Classification Methods}

Classification methods identify signal vs. background events based upon a set of features (also called variables, attributes, or scores) which describe the events. For example, objects in photometric images can be described by their magnitude, signal-to-noise, and shape parameters such as width and ellipticity. These features can be used to distinguish stars from galaxies, cosmic rays, or imaging artifacts.

The optimum separation of two classes of events is application dependent, depending upon the desired tradeoff between purity (the fraction of selected events which are real 
signal), completeness (the fraction of real signal events which are selected), and the total sample size selected. For example, a measurement which depends upon a statistical fit to

both signal and background events might optimize the signal-to-noise ratio $\sim S / \sqrt{S+B}$ where $S$ and $B$ are the number of real signal and background events which the classifier selects for the fit. A supernova search algorithm, on the other hand, might maximize the purity with the constraint that the completeness remain above $90 \%$.

Some methods, such as threshold cuts (\$2.1), produce a boolean signal/background decision and the cuts themselves must be adjusted to optimize the separation. Other methods have an automated training procedure and produce a single statistic which rates how signallike or background-like a new event is. The user may then cut on that statistic to optimize the desired figure of merit.

Classifier parameters are tuned using a training dataset of known signal and background events to optimize the separation power. Since the results are influenced by the particular statistical fluctuations of the training dataset, the separation power on the training data itself cannot be used as a fair measure of the power of a classifier. Instead, a separate validation set is used to assess the performance. If enough training data is available, one uses one dataset to train a variety of classifiers with different parameters, a second dataset to select which set of parameters produces the best classifier, and a third dataset to validate the final performance. Ideally one trains and validates using real data; in practice simulated data are often used for training and validation before applying the classifier to real data. It is important to note that the quality and power of any classifier will be affected by the accuracy of the training sample. One must be careful to minimize and measure any biases introduced through a simulated training sample which does not completely reflect real data.

\subsection{Threshold Cuts}

Automated supernova searches have typically operated by applying simple threshold cuts to the features describing objects. For example, a supernova search might keep objects which have a signal-to-noise ratio $S / N>5$, astrometric positions that agree to within 1 arcsec on 2 or more images, and a width consistent with stars on the images (e.g., within a factor of 2 of the median width of stars). If an object fails any of these cuts, it is rejected. These cuts are easy to understand but do not reflect the subtleties of a multidimensional space. An object which just barely fails one of the cuts is still rejected the same as an object which fails many cuts. It also does not naturally handle correlations between the variables, 
e.g., between the $S / N$ and the astrometric accuracy 5 To use threshold cuts, one must find uncorrelated variables without significant outliers such that every cut maintains a high signal efficiency while rejecting background.

Compared to curved boundary selections, threshold cuts are also an inefficient way to select a subset of a hyperspace as the number of dimensions grows large (Köppen 2000), even for dimensions as few as 5. e.g., for 3 dimensions, the volume ratio of a cube to its embedded sphere is 1.9; for 5 dimensions it is 6.1 , and for 10 dimensions it is 401.5 . This ratio goes to infinity as the number of dimensions increases. Thus if a set of signal events is distributed as an ellipsoid in some feature space, an ellipsoid shaped selection contains much less volume (and thus likely much less background) than the equivalently dimensioned hypercube.

Although commonly used in supernova searches, threshold cuts are widely recognized as being a non-optimal method for signal/background separation problems. The following sections describe a variety of more powerful techniques for identifying supernovae in difference images.

\subsection{Multi-dimensional Probability Measures}

A more sophisticated approach models the probability distribution function (PDF) for the signal and the background for each of the features. The combined probability of all of the feature values for an object is used to make the signal/background decision. This improves over threshold cuts by eliminating rejections based upon a slightly marginal value of a single feature, but it requires a detailed modeling of the PDF of each feature, including all correlations and outliers in the distributions. This suffers from the "curse of dimensionality" (Bellman 1961): since the volume of a hyperspace grows exponentially with the number of dimensions, the size of a training sample must also grow exponentially to adequately determine the PDFs.

If the signal and background features are Gaussian distributed with only linear correlations, Fisher Discriminant Analysis (Fisher 1936) finds the best linear combination of features to maximize the separation of the two classes. Figure 1 shows a toy example of data which would be well separated using Fisher Discriminant Analysis. The two classes of events (blue triangles and red squares) are not well separated by either feature $A$ or $B$, but their correlation is such that the combination $A+B$ provides very good separation of the

\footnotetext{
${ }^{5}$ In a simple case such as this, one could combine $S / N$ and astrometric positions to form an uncorrelated variable; accomplishing this in the general case for a large number of variables is non-trivial.
} 
two classes.

More generally, if a set of events $\{\mathbf{x}\}$ in some feature space have means $\boldsymbol{\mu}_{0,1}$ and covariances $\Sigma_{0,1}$ for classes 0 and 1 , then a linear combination $\mathbf{w} \cdot \mathbf{x}$ will have means $\mathbf{w} \cdot \boldsymbol{\mu}_{0,1}$ and covariances $\mathbf{w}^{T} \Sigma_{0,1} \mathbf{w}$, where $\mathbf{w}$ is a set of coefficients defining a linear combination of the features. The separation of the two classes may be defined as

$$
\Delta=\frac{\left(\mathbf{w} \cdot \boldsymbol{\mu}_{0}-\mathbf{w} \cdot \boldsymbol{\mu}_{1}\right)^{2}}{\mathbf{w}^{T} \Sigma_{0} \mathbf{w}+\mathbf{w}^{T} \Sigma_{1} \mathbf{w}}
$$

i.e., the separation of the means is measured in units of the variances. Fisher showed that the maximum separation is achieved when

$$
\mathbf{w}=\left(\Sigma_{0}+\Sigma_{1}\right)^{-1}\left(\boldsymbol{\mu}_{1}-\boldsymbol{\mu}_{0}\right)
$$

The means and covariances of the signal (1) and background (0) classes may be estimated from a training sample, and thus the calculation of the best linear combination for separating the classes is simply a matrix inversion. This method breaks down when there are non-linear correlations or when there are significant outliers or otherwise non-Gaussian variances such that a simple mean and covariance is not a good descriptor of the feature distributions for the two classes. In practice, Fisher Discriminant Analysis is most often used to combine several linearly correlated features into a single feature to reduce the dimensionality of a problem before applying another classification method.

\subsection{Decision Trees}

Decision trees (Breiman et al. 1984) separate signal from background events by making a cascading set of event splits as shown in Figure 3. This forms a generalization of threshold cuts by selecting many hypercubes in the multi-dimensional feature space rather than a single hypercube of cuts. The training procedure described below automatically selects the features and cut values to generate a tree with maximal separation of signal and background events.

The training procedure begins with a sample of training events and considers all features and cut values to form two subsets with the best separation of signal and background. The procedure is recursively applied to each of the subsets to form further branches. The recursion is stopped when some condition is met, e.g., the subset is entirely signal or background, or the subset has reached a minimum allowed size (a minimum size requirement prevents overtraining on statistical fluctuations of small samples). The terminal nodes which are not further split are called leaves, and are assigned as either signal or background leaves depending upon the training events which ended up on those leaves. 
There are a variety of ways to define the best separation at each split; for this study we used the Gini parameter (Gini 1921; Breiman et al. 1984), which is widely used and provides robust performance. Define the purity of a sample of training events as

$$
P=\frac{\sum_{S} w_{S}}{\sum_{S} w_{S}+\sum_{B} w_{B}}
$$

where the sums are over the signal events $S$ and background events $B$ and $w_{i}$ are a set of event weights. Typically all of the weights are the same and their absolute normalization is arbitrary. If needed, relative weights may be used to increase the influence of an underrepresented subsample of the training data. The role of weights will be more important in the Boosted Trees method described in $\$ 2.3 .1$. Note that $P=1$ for a sample of pure signal events, $P=0$ for a sample of pure background events, and $P(1-P)=0$ for a sample which is either purely signal or purely background.

Define

$$
\text { Gini }=P(1-P) \sum_{i=1}^{n} w_{i}
$$

where the sum is over all events in that sample. At each node, the training procedure considers all possible features and cut values to minimize the quantity

$$
\text { Gini }_{\text {left child }}+\text { Gini }_{\text {right child }}
$$

to find the best separation of events. If this split would not increase the overall quality of the tree, i.e.,

$$
\text { Gini }_{\text {parent }}<\text { Gini }_{\text {left child }}+\text { Gini }_{\text {right child }}
$$

then the node is left as a leaf node, assigning it as a signal leaf if $P>0.5$ and a background leaf otherwise. If the split would increase the overall quality of the tree, the events are split into two nodes and the procedure is recursively applied to each of those nodes until the stopping conditions are met (e.g., minimum leaf sizes) or no splits can be found which would improve the overall quality of the tree.

Decision trees are a generalization of threshold cuts and thus have more flexibility to optimally select a set of signal events within a feature space. However, single decision trees tend to be unstably dependent upon the details of the training set. A small change in the training set can produce a considerably different tree and thus a considerably different performance on the validation set. 


\subsubsection{Boosted Trees}

Boosting algorithms improve the performance of a classifier by giving greater weight to events which are hardest to classify. In the case of decision trees, a tree is trained on a set of data, misclassified events are identified and their weights are increased, and the process is repeated to form new trees. This iteratively produces a set of increasing quality decision trees. The final classifier uses the weighted ensemble average of all of the trees to make a classification decision. The boosting provides decision trees with better separation power, and the ensemble average washes out the training instabilities associated with single decision trees. In applications with $\sim 20$ or more input features, Boosted Decision Trees can provide significantly better results than Artificial Neural Networks (Roe et al. 2005); see also \$2.5.

There are a variety of boosting algorithms used to increase the weights of misclassified events (Freund \& Schapire 1996; Friedman 2001; Friedman, Hastie, \& Tibshirani 2000). We describe here the commonly used Discrete AdaBoost method (Freund \& Schapire 1996). Define the error rate for tree $m$ as

$$
\operatorname{err}_{m}=\frac{\sum_{i=1}^{N} w_{i} I_{i}}{\sum_{i=1}^{N} w_{i}}
$$

where $I_{i}=0$ if event $i$ is correctly classified and $I_{i}=1$ if it is incorrectly classified. Typically the first tree is trained with the same weight for all events. Then adjust each of the event weights using

$$
\begin{aligned}
\alpha_{m} & =\beta \times \ln \left[\left(1-\operatorname{err}_{m}\right) / \operatorname{err}_{m}\right] \\
w_{i} & \rightarrow w_{i} \times e^{\alpha_{m} I_{i}}
\end{aligned}
$$

This increases the weights of misclassified events; the weights are increased more when the tree has a low error rate. These new weights are then used to generate a new decision tree. The standard AdaBoost algorithm uses $\beta=1$ but this can be adjusted to vary how quickly the weights are updated with each iteration.

After generating $M$ individual trees with weights $\alpha_{m}$, the final classifier answer for an event described by a set of features $\mathbf{x}$ is

$$
T(\mathbf{x})=\sum_{m=1}^{M} \alpha_{m} T_{m}(\mathbf{x})
$$

where $T_{m}(\mathbf{x})$ is the result for tree $m$ : 0 if $\mathbf{x}$ lands on a background leaf and +1 for a signal leaf. The absolute normalization of $T(\mathbf{x})$ is arbitrary; we chose to renormalize the $\alpha_{m}$ weights such that $0 \leq T(\mathbf{x}) \leq 1$. 


\subsubsection{Random Forests}

Random Forests (Breiman 2001) also generate multiple decision trees for a given training set and use a weighted average of the trees as the final decision metric. When training a tree, at each branch the training cycle only considers a random subset of the possible features available to use. This has the effect of washing out the typical training instabilities of decision trees and produces a classifier which is fast to train and robust against outliers.

\subsection{Support Vector Machines}

The Support Vector Machine (SVM) algorithm is a classification method that has successfully been applied to many pattern recognition problems and is founded on principles of statistical learning theory (Vapnik 1998; Chen et al. 2005). It nonlinearly maps data points from the original input space to a higher-dimensional feature space in which an optimal hyperplane parameterized by a normal vector $\mathbf{w}$ and offset $b$ is computed such that the separation between events in different classes is maximized. The linear decision boundary is defined as $f(\mathbf{x})=\mathbf{w} \cdot \phi(\mathbf{x})+b$, where $\mathbf{x}$ is a vector in the feature space which describes objects and $\phi$ is a mapping which embeds the problem into a higher-dimensional space in which classes are more easily separable than in the original feature space.

An optimization problem is constructed to find the unknown hyperplane parameters, and the optimal hyperplane normal $\mathbf{w}$ is found to be entirely determined by the subset of events nearest to the optimal decision boundary (also called support vectors, $\mathbf{x}_{i}$ ) as follows: $\mathbf{w}=\sum_{i} c_{i} \phi\left(\mathbf{x}_{i}\right)$, where the coefficients $c_{i}$ are the Lagrange multipliers used in solving the nonlinear optimization and are a byproduct of the optimization.

The hyperplane parameters are solved by maximizing the margin (the distance between the hyperplane and the example events in each class), which is formulated as a nonlinear constrained optimization problem, where the constraints enforce that examples from different classes lie on opposite sides of the hyperplane. The objective function to be minimized is convex, i.e., it is guaranteed to have a global minimum and no local minima. The linear decision boundary corresponds to a nonlinear (and possibly disjoint) decision boundary in the original feature space. Once the hyperplane is found, a set of features $\mathbf{x}$ is typically classified into one of the two classes by applying a threshold cut to $f(\mathbf{x})$.

Rather than calculating $\phi(\mathbf{x})$ explicitly while evaluating $\mathbf{w} \cdot \phi(\mathbf{x})=\sum_{i} c_{i} \phi\left(\mathbf{x}_{i}\right) \cdot \phi(\mathbf{x})$, the actual embedding is achieved through a kernel function defining an inner product in the embedding space, $k\left(\mathbf{x}_{1}, \mathbf{x}_{2}\right)=\phi\left(\mathbf{x}_{1}\right) \cdot \phi\left(\mathbf{x}_{2}\right)$. This "kernel trick" makes class prediction easy to implement and fast to compute. Several common kernel mappings are given in Chen et al. 
(2005). In practice, the kernel function is typically chosen empirically via training and testing, and the simplest function giving the desired performance is used. The Gaussian kernel used in this analysis

$$
k\left(\mathbf{x}_{1}, \mathbf{x}_{2}\right)=\exp \left(-\left\|\mathbf{x}_{1}-\mathbf{x}_{2}\right\|^{2} / 2 \sigma^{2}\right)
$$

is commonly used because it only has one free parameter to be tuned $(\sigma)$ and empirically performs as well as, if not better than, more complex kernels which may overfit the data.

For this analysis we used a soft-boundary SVM method called $C$-SVM, which handles noisy data with high class overlap by adding a regularization term to the objective function. This term allows but penalizes training points lying on the wrong side of the decision boundary. The regularization parameter, $C$, controls the trade-off between maximizing the separation and allowing some amount of training error while finding the hyperplane which maximally separates signal from background.

The advantages of SVMs include the existence of a unique solution, the simple geometric interpretation of the margin maximization function, the capacity to compute arbitrary nonlinear decision boundaries while controlling over-fitting with soft margins, the low number of parameters to be tuned (as few as two, depending on the choice of kernel), and the dependence of the solution on only a small number of data points (the support vectors) which define the boundary of the class separation hypersurface. For SVM implementation details, see Vapnik (1998).

\subsection{Artificial Neural Networks}

Artificial Neural Networks (ANNs) are a broad category of classification methods originally inspired by the interconnected structure of neurons and synapses in the brain. These methods map a set of input variables to one or more output results via one or more "hidden layers" of intermediate nodes. For a supernova search, the inputs would be the features describing each object and the desired output would be 1(0) for signal (background). For an overview of these methods, see Bishop (1996).

ANNs can be powerful classifiers and have been used in many applications, though they are slow to train and require some experimentation to optimize the number of hidden layers and nodes to match a given problem. They also do not scale well with an increasing number of input features, and their results become unstable when there are significant outliers or otherwise irrelevant input data. For these reasons, ANNs were not deemed to be an appropriate classification method for our dataset and this method was not pursued for this study. 


\section{Nearby Supernova Factory Search}

The Nearby Supernova Factory (SNfactory, Aldering et al. 2002) search uses data from the Near Earth Asteroid Tracking (NEAT) program 6 and the Palomar QUEST consortium 7 using the 112 CCD QUEST-II camera (Baltay et al. 2007) on the Palomar Oschin 1.2-m telescope. The NEAT observing pattern obtains triplets of 60-second exposures spread over a time period of $\sim 1$ hour using a single RG610 filter, which is a long pass filter redward of $610 \mathrm{~nm}$. This allows the search to distinguish between asteroids, whose motion is typically detectable on that timescale, and spatially static objects such as supernovae. The QUEST data are obtained in 4 filters in driftscan mode; our search uses the two filters which cover the best quality CCDs (either Bessel $R$ and $I$ or Gunn $r$ and $i$ depending upon camera configuration). The QUEST data cover less area and tend to be cosmetically cleaner than the NEAT data, resulting in fewer spurious detections overall. Since the false positive background events are much worse in the NEAT data, our study of alternative classification methods has focused on the NEAT dataset.

Coadded stacks of images taken from 2000 to 2003 are used as references. The new and reference images are convolved to match their point-spread-functions (PSFs), the fluxes are normalized by matching stars, and the reference is subtracted from the new images. Objects in the subtraction are identified based upon contiguous pixels with $S / N>3$ with at least one pixel with $S / N>5$. Objects are described by features such as position, fullwidth-half-max (FWHM) in $x$ and $y$, aperture photometry and associated uncertainties in 3 apertures, distance to nearest object in the reference coadd, and measures of the roundness and irregularity of the object contour based upon Fourier descriptors (Zahn \& Roskies 1972). Additional features are formed as combinations of features from the same object observed on multiple images. Combined features include the object motion between two images and the consistency of the statistical significance of the measurements in different images. The features are used by a classification method (originally threshold cuts, more recently Boosted Decision Trees) to select supernova candidates of interest which are then visually scanned by humans to select the best candidates for spectroscopic confirmation and followup by the SuperNova Integral Field Spectrometer (SNIFS) (SNfactory, Aldering et al. 2002), on the University of Hawaii 2.2-m telescope on Mauna Kea.

\footnotetext{
${ }^{6}$ http://neat.jpl.nasa.gov

${ }^{7}$ http://hepwww.physics.yale.edu/quest/palomar.html
} 


\section{Training Dataset}

To generate signal events for training, fake supernovae were introduced into the images by moving real stars of a desired magnitude to locations distributed about known galaxies on the SNfactory search images. By using real stars from the same images as the galaxies, we realistically model the point-spread-function, noise, and possible image artifacts present in that image. These images with fake supernovae were processed with the same data analysis pipeline as real images to identify objects and measure their features for classification.

The stars are sampled in a circular region of 20 pixels in diameter. The typical FWHM of stars on these images is about 3 pixels, so this samples the PSF out to $\sim 8 \sigma$. The average sky level of the image is subtracted from the sampled pixels before they are added in the new location, which implicitely assumes that the sky level is uniform over the image. This is valid in most cases, and it is simple to reject fakes created from cases that violate this assumption. Typically these fakes will have a FWHM that differs very significantly from the average $(\sim 20$ pixels vs. $\sim 3)$. Most stars are sufficiently isolated that they do not bring along portions of other objects, and we identify and reject cases where this does happen. The spatial variation of the PSF is minimal compared to the night-to-night variations which much be addressed by the image subtraction pipeline, thus this fake supernova generation procedure does not attempt to correct for the small spatial variations of the PSF across the CCD.

Background events were randomly selected from 4.1 million other objects identified on the subtractions with fake supernovae. These subtractions covered a month of data taking including bright and dark times and a variety of seeing conditions. Objects within 20 pixels of a fake supernova were excluded to avoid any artifacts which might be introduced through an ill-formed fake. These background events form a randomly selected subset of the genuine backgrounds faced by the supernova search in the real data, and thus represent the real fractions of each type of background event faced.

The signal and background samples were split into training and validation subsets. Several training sets were formed with 5,000 signal and 5,000 background events each. The final validation was performed using 20,000 signal and 200,000 background events. The training dataset for the Support Vector Machine method was augmented with real supernova discoveries in an attempt to improve its overall performance. The original training set used 19 features; an additional 13 features were then added which improved the performance of the Boosted Trees and Random Forests but decreased the performance of the SVM. The results shown in $\$[6$ are for the best performance achieved for each classifier (i.e., using 19 features for SVM and 32 features for the other methods). 


\section{Classification software}

For Fisher Discriminant Analysis, Boosted Trees, and Random Forests, we used the open-source $\mathrm{C}++$ software package StatPatternRecognition 8 Training a set of 200 boosted trees using 10,000 training events with 19 features each with a minimum leaf size of 15 events 9 takes $\sim 3$ minutes (wallclock) on a $2 \mathrm{GHz}$ AMD Opteron CPU. Training with 32 features takes $\sim 45$ minutes. Once the trees are trained, it takes approximately $0.6 \mathrm{~ms}$ (wallclock) to evaluate the results for an object. Random Forests took less than 2 minutes to train on the dataset with 32 features, using the same parameters as above. Evaluation of a new event takes approximately $0.2 \mathrm{~ms}$. Fisher Discriminant analysis took 1 second to train on the 32 feature dataset and $0.07 \mathrm{~ms}$ to evaluate results for a new object.

For SVM, we used the LIBSVM C++ package 10 Training a $C$-SVM using 10,000 training events with 19 features each takes from 5 to 15 seconds (wallclock) on a $2 \mathrm{GHz}$ AMD Opteron CPU, depending on the settings of the two parameters used (if the parameters overfit the data, more support vectors are needed so training time increases). Evaluating the SVM on a new data point takes approximately $0.6 \mathrm{~ms}$ (wallclock).

\section{Comparison of Methods}

In the end, most classification methods produce a single classification statistic with arbitrary normalization which rates how signal-like or how background-like the candidate is. A threshold cut on this statistic can be used to select a subsample of events with desired signal vs. background purity. A useful way to visualize the power of a classifier is to plot the fraction of false positives (i.e., background events incorrectly classified as signal) vs. fraction of true positives (i.e., signal events correctly identified) for various selection values on the classification statistic.

Figure 4 shows the performance of several classification methods applied to the SNfactory dataset. The red square shows the performance of the original threshold cuts upon which we were working to improve. The curves show that SVM, Random Forests, and Boosted Trees all performed dramatically better than the threshold cuts across a wide range of signal and background efficiencies. The object features in our data have significant outliers which

\footnotetext{
8 http://sourceforge.net/projects/statpatrec

${ }^{9}$ The selection of the number of trees and minimum leaf size are described in section 6.1

10 http://www.csie.ntu.edu.tw/ cjlin/libsvm
} 
prevented Fisher Discriminant Analysis from being a useful classification method.

The overall best performance was obtained using Boosted Decision Trees, with Random Forests providing nearly as good performance with faster training and evaluation times. Although SVM performed considerably better than threshold cuts, it was not as successful as Random Forests or Boosted Trees. A possible explanation is that the SVM proved to be more sensitive to signal events that lie close to background events in the feature space, and could not strike a balance between modeling such events vs. overfitting to noise. For example, a dim young supernova on a bright galaxy can be very similar to a statistical fluctuation or a modest subtraction error in the images. Robustness against overfitting is a known strength of boosted classifiers (Freund \& Schapire 1999), and the issue of overfitting noisy data is an area of active research within the machine learning community. Further details of applying SVM to the SNfactory dataset are described in Romano et al. (2006).

Boosted Trees, Random Forests, and SVM successfully reduced the false-positive rate for all types of background events in our data. The three most common remaining background types are faint optical ghosts from scattered light, fluctuations in charge trails from bright stars due to CCD charge transfer inefficiency, and leftover dipoles from subtracting astrometrically misaligned objects. The optical ghosts can be genuinely difficult to distinguish from dim supernovae near our detection threshold. The charge trails and dipoles are easy to distinguish by eye but we currently do not have specific features which directly address these two backgrounds, thus all classification methods have difficulty with them, given the input features currently available. These backgrounds are somewhat described by a roundness feature and comparison of the flux in small vs. large apertures. Adding features to directly address these backgrounds would improve the power of any classification method. Projects with higher quality CCDs and optical designs to minimize scattered light will naturally have fewer backgrounds as well.

The optimum selection criterion is dependent upon the tradeoff between true positive selection efficiency (horizontal axis) and the false positive selection efficiency (vertical axis). At the SNfactory we seek to maximize our signal efficiency within the realistic constraints of the personnel and telescope time available to vet false positives. For the Fall 2006 search, we used Boosted Decision Trees and choose a point with 10 times less background than we had previously faced. This corresponds to an average efficiency of $\sim 78 \%$ for identification of a supernova with a single filter and one night of imaging. 


\subsection{Optimizing Boosted Tree Parameters}

Since Boosted Trees provided the best classification performance, we describe here how the performance changed with various input parameters to the Boosted Tree training. The

performance of Boosted Trees depends upon the number of trees generated and the amount of branching which is done before finishing each tree.

For controlling the amount of branching per tree, the StatPatternRecognition package has an adjustable limit on minimum number of events per leaf in the final tree. Our training sample contained 5,000 signal and 5,000 background events. We found the best performance with a minimum leaf size of 15 events, which results in a set of boosted trees with 250-300 leaves each. Figure 5 shows the relative performance of 200 trees with a mininum leaf size of $n=5,25,50,100$ relative to the $n=15$ case.

Figure 6 shows the relative performance of $N=25,50,100,200$ trees in comparison to the $N=400$ case, using a minimum leaf size of 50 events. For the Fall 2006 SNfactory supernova search we choose to use 200 trees with a minimum of 15 events per leaf out of the 10,000 training events.

\section{Combining Methods}

As expected, the various methods provided correlated output; i.e., events ranked highly by one classifier tended to be ranked highly by another. But even through Boosted Trees provided the best classification performance overall, there were good signal events which were found by SVM which were missed by the Boosted Trees (for a given set of thresholds on the SVM and Boosted Tree outputs). We attempted to recover these events by combining the output of these two methods. Several combinations were tried:

- Keep events which passed thresholds for either classifier.

- Perform Fisher Discriminant Analysis on the output of the two classifiers.

- Split the SVM vs. Boosted Tree output space into sub-regions of signal and background. This is conceptually similar to forming a decision tree in this $2 \mathrm{D}$ space and accounts for non-linear correlations in the SVM vs. Boosted Tree output.

- Use the output of SVM as an additional feature input for Boosted Trees.

None of these methods produced results which outperformed the Boosted Trees alone. Although the combined classifiers could identify signal events which would have been missed 
by just one classifier, the combination also brought in an increased number of background events such that the overall performance was the same or worse than the Boosted Trees alone. This result is not generally true, however. In other contexts, multiple classifiers have been successfully combined to produce overall more powerful results (Dietterich 2002; Kittler et al. 1998).

\section{Discussion and Conclusion}

This work has shown a variety of object classification methods which provide significantly better performance than is possible with the method of threshold cuts used by most current supernova searches. The implementations studied here used common defaults, such as using the Gini parameter for optimizing Boosted Decision Trees and the Gaussian kernel for SVM. There are many variations of these methods, some of which might provide further improved performance. But even these "out of the box" implementations with minimal tuning provided much better performance than threshold cuts.

Any classifier will be limited by the quality and power of the input features provided. In practice, after an initial round of training and validation, one should study the misclassified events and introduce additional features that distinguish these. Such iterations are helpful regardless of which classification method is used; the main point of this work is to point to new classification methods which will maximize the classification power possible given a set of features.

As with any analysis, there is no substitute for clean data and a well understood detector. Problems which arise from false-positive detections should first be addressed at the level of the detector and data processing pipeline. Future projects will hopefully have the resources to address spurious detections at this level to make the process easier for their object classifiers. But even high quality, well understood detectors and advanced image processing pipelines such as SDSS will face signal vs. background classification problems, and this is where the methods described in this paper come into play.

In addition to improved background rejection power, these new methods also have the advantage of generating a single number which ranks the quality of an object rather than a boolean pass/fail decision. One may then adjust a threshold cut on that single number to tune the desired tradeoff between purity and completeness. Future surveys may publish transient alerts using relatively loose quality requirements; subscribers to these alerts can then place their own cuts on this quality rank to adjust the purity, completeness, and input data rate as needed. 
Boosted Trees, Random Forests, and Support Vector Machines all provide much better object classification performance than traditional threshold cuts. When applied to the SNfactory supernova search pipeline, Boosted Trees enabled us to find more supernovae with less work: Our efficiency for finding real supernovae increased while our workload for scanning non-supernova objects dramatically decreased. Methods such as these will be crucial for maintaining reasonable false positive rates at the automated transient alert pipelines of upcoming projects such as PanSTARRS and LSST.

We would like to thank G. Aldering, S. Bongard, M.J. Childress, P. Nugent, and R. Scalzo for useful conversations and assistance with scanning our supernova candidates. We also thank the entire Nearby Supernova Factory collaboration for confirmation and followup spectra of our selected candidates and for the use of the search images for this study. The anonymous referee provided many useful comments for which we are grateful.

We are grateful to the technical and scientific staff of the Palomar Oschin telescope, where our supernova search data are obtained. The High Performance Wireless Research and Education Network (HPWREN) 11 , funded by the National Science Foundation grants 0087344 and 0426879 , has provided a consistently reliable network for transferring our large amount of data from Mt. Palomar in a timely manner.

This work was supported in part by the Director, Office of Science, Office of High Energy and Nuclear Physics, of the U.S. Department of Energy under Contract No. DE-FG0292ER40704, by a grant from the Gordon \& Betty Moore Foundation, by National Science Foundation Grant Number AST-0407297. This research used resources of the National Energy Research Scientific Computing Center, which is supported by the Office of Science of the U.S. Department of Energy under Contract No. DE-AC02-05CH11231.

SB would especially like to thank the organizers and hosts of the Statistical Inference Problems in High Energy Physics and Astronomy Workshop held at the Banff International Research Station (BIRS), which is supported by the U.S. National Science Foundation, the Natural Science and Engineering Research Council of Canada, Alberta Innovation, and Mexico's National Council for Science and Technology (CONACYT).

Facilities: PO:1.2m (QUEST-II)

\footnotetext{
${ }^{11}$ http://hpwren.ucsd.edu
} 


\section{REFERENCES}

Aldering, G. et al. (SNfactory) 2002, Proc. SPIE, 4836, 61

Astier, P. et al. (SNLS) 2006, A\&A, 447, 31-48

Baltay, C. et al. 2007, (astro-ph/0702590)

Becker, A. et al. (SDSS-II Supernova Survey) 2007, BAAS (Seattle, WA)

Bellman, R.E. 1961, Adaptive Control Processes (Princeton, NJ: Princeton University Press)

Bishop C.M. 1996, Neural Networks for Pattern Recognition (Oxford University Press)

Breiman, L. 2001, "Random Forests," University of California, Berkeley, technical report

Breiman, L., et al. 1984, Classification and Regression Trees (Belmont, CA: Wadsworth International Group)

Chen, P.-H., Lin, C.-J., and Scholkopf, B. 2005, Applied Stochastic Models in Business and Industry, 21(2), 111-136

Dietterich, T.G. 2002, Ensemble Learning, in The Handbook of Brain Theory and Neural Networks, Second Edition (M.A. Arbib, Ed.) (Cambridge, MA: The MIT Press)

Fisher, R.A. 1936, Annals of Eugenics, 7, 179-188

Freund, Y., and Schapire, R.E. 1996, Proc COLT, 209-217 (New York: ACM Press)

Freund, Y., and Schapire, R.E. 1999, J. Japan. Soc. for Artif. Intel. 14(5), 771-780

Friedman, J. 2001, Annals of Statistics 29(5), 1189-1232

Friedman, J., Hastie, T., Tibshirani, R. 2000, Annals of Statistics, 28(2), 337-407

Gini, C. 1921, The Economic Journal 31, 124-126

Kittler, J. et al. 1998, IEEE Trans. Pattern Analysis and Machine Intelligence, 20(3), 226

Köppen, M. 2000, 5th Online World Conference on Soft Computing in Industrial Applications (WSC5), held on the Internet, https://www.npt.nuwc.navy.mil/Csf/papers/hidim.pdf

Miknaitis et al. (ESSENCE) 2007, (astro-ph/0701043), ApJ, submitted

Roe, B.P. et al. 2005, Nucl. Instrum. Meth. A543, 277-584 
Romano, R., Aragon, C., Ding, C. 2006, in Proceedings of the 5th International Conference of Machine Learning Applications (Orlando, FL: IEEE)

Vapnik, V. 1998, Statistical Learning Theory (Wiley)

Zahn, C.T., and Roskies, R.Z. 1972, IEEE Trans. Computers, 21, 269-281 


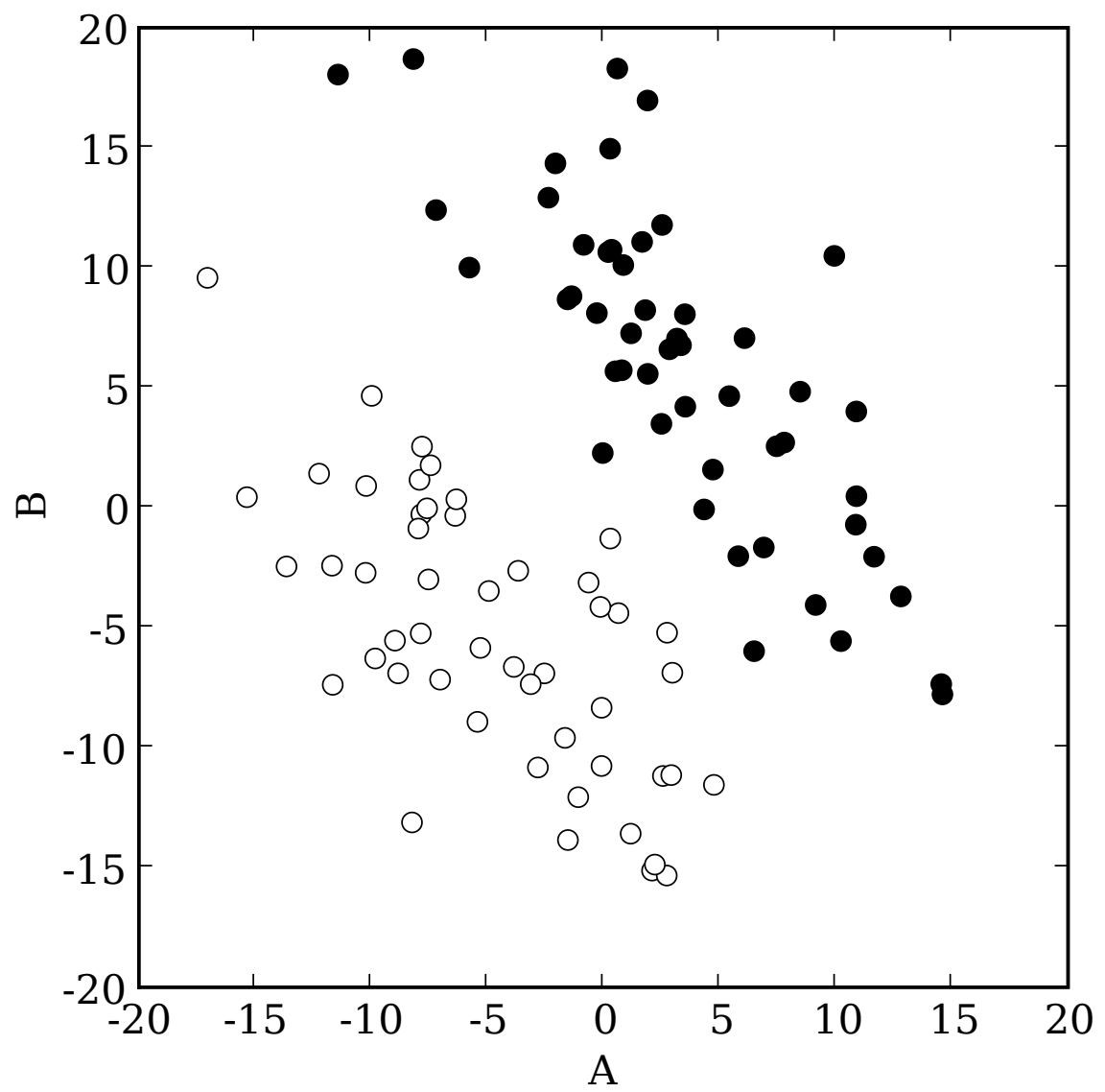

Fig. 1.- Example data which would be well separated using Fisher Discriminant Analysis. The two classes of events (open and filled circles) are not well separated by either feature $A$ or $B$, but their correlation is such that the combination $A+B$ provides very good separation of the two classes. 

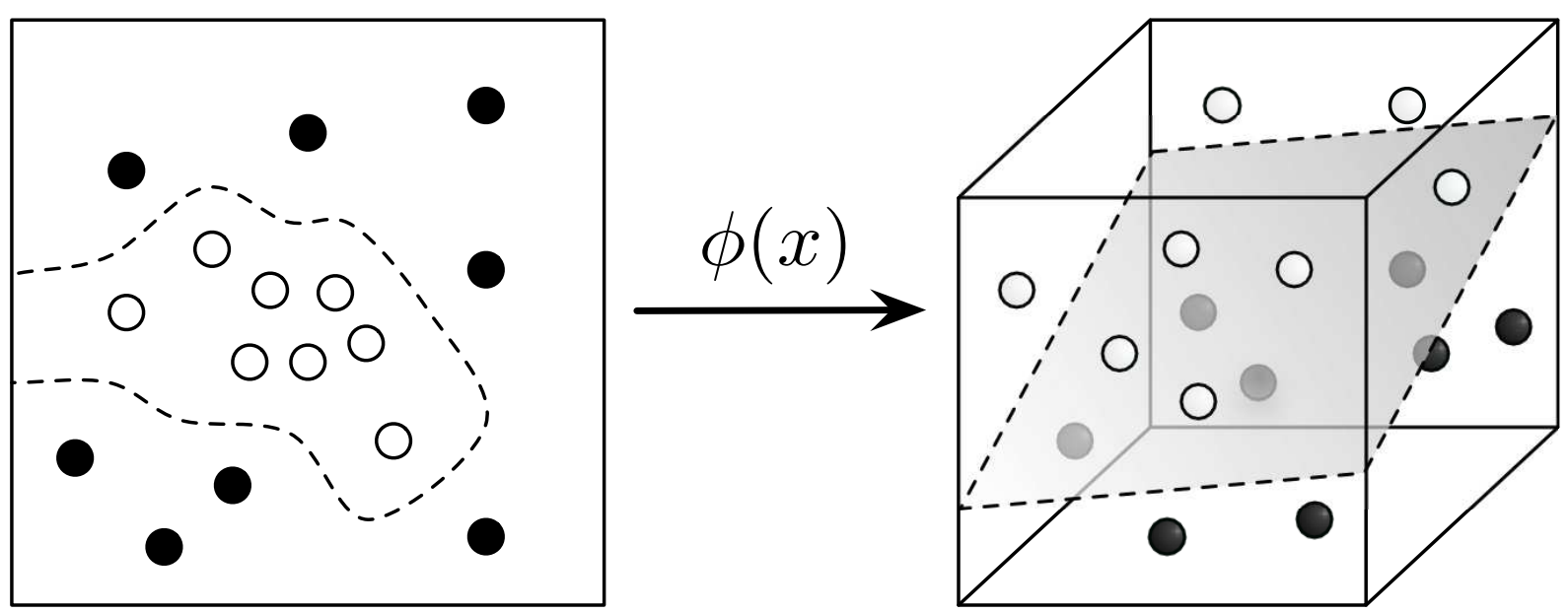

Fig. 2.- Support Vector Machines map an input space of features into a higher dimensional space where the separation of classes becomes easier. The separation boundary in the original space may be quite complex, even disjoint. In the higher dimensional space, the separation surface is a hyperplane whose parameters are entirely determined by the subset of events (the support vectors) nearest to the boundary. 


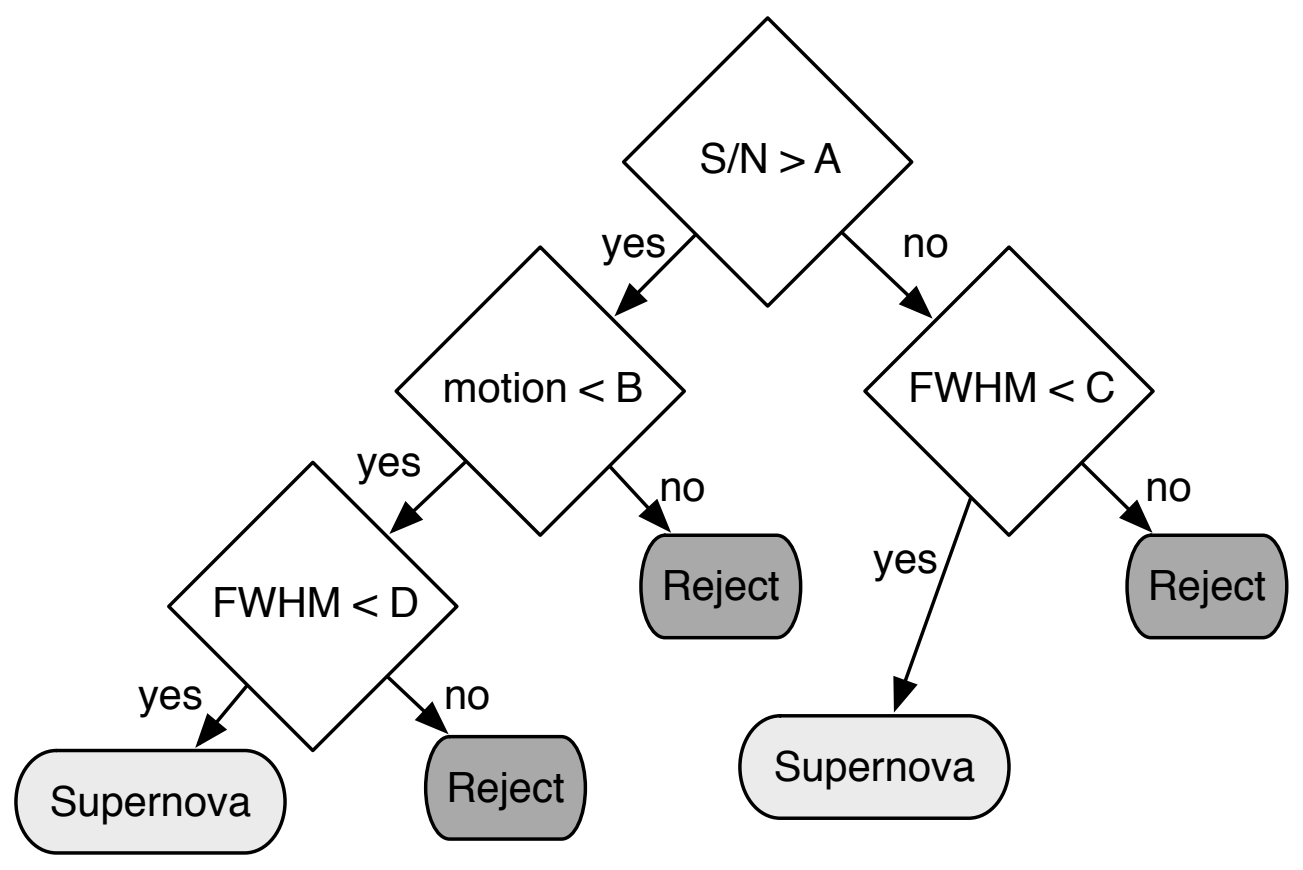

Fig. 3.- Example decision tree which would treat high signal-to-noise objects differently than low signal-to-noise objects. In practice, a real decision tree has many more branches and the same variable can be used to branch at many different locations with different cut values. 


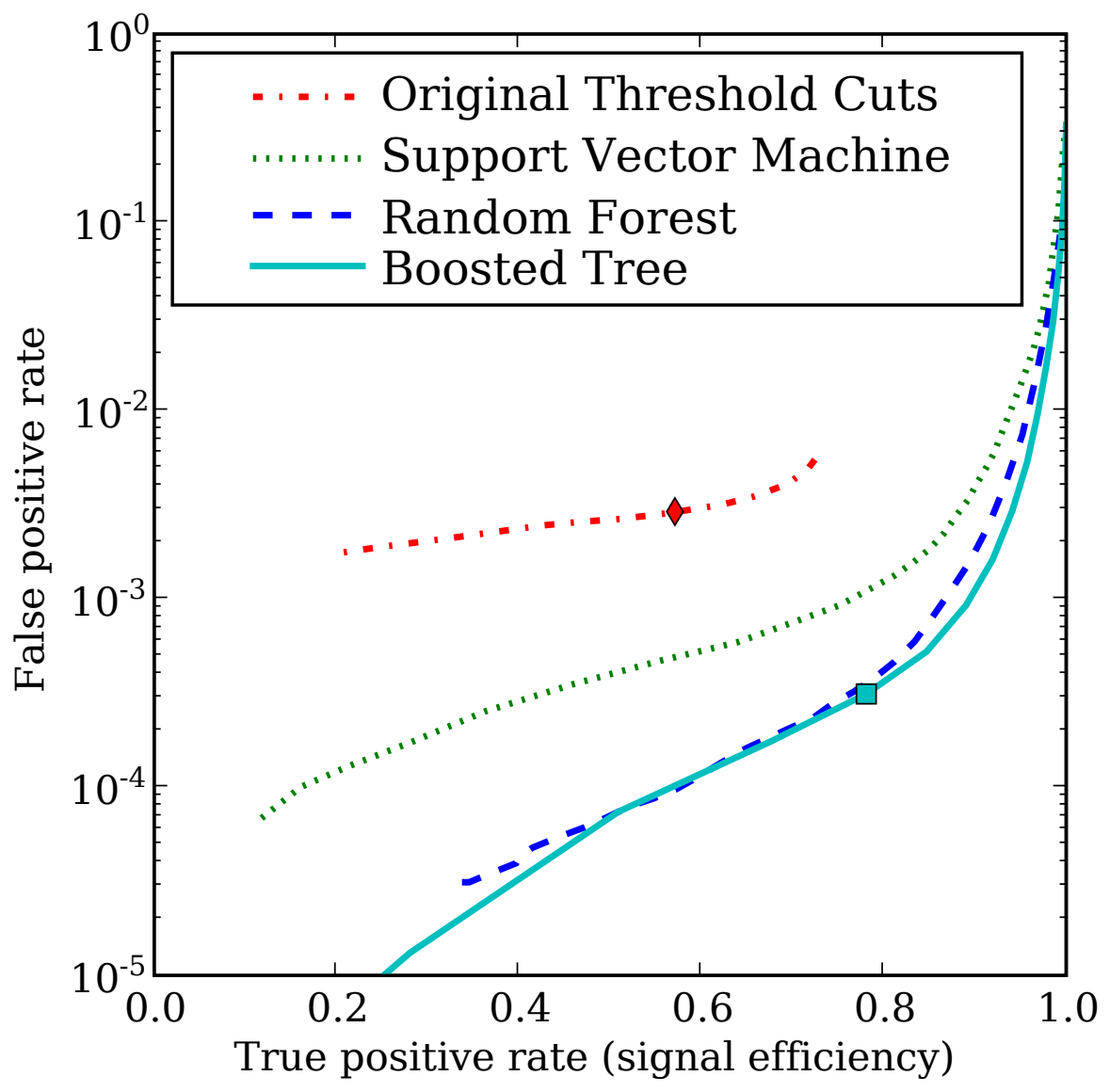

Fig. 4.- Comparison of Boosted Trees (cyan solid line), Random Forest (blue dashed line), SVM (green dotted line), and threshold cuts (red dash-dotted line) for false positive identification fraction vs. true positive identification fraction. For the threshold cuts, the signal-to-noise ratio, motion, and shape cuts were varied to adjust signal and background rates. The red diamond shows the performance of the threshold cuts used during the SNfactory Summer 2006 search; the cyan square shows the performance achieved with Boosted Trees which were used for the Fall 2006 SNfactory search. The lower right corner of the plot represents ideal performance. 


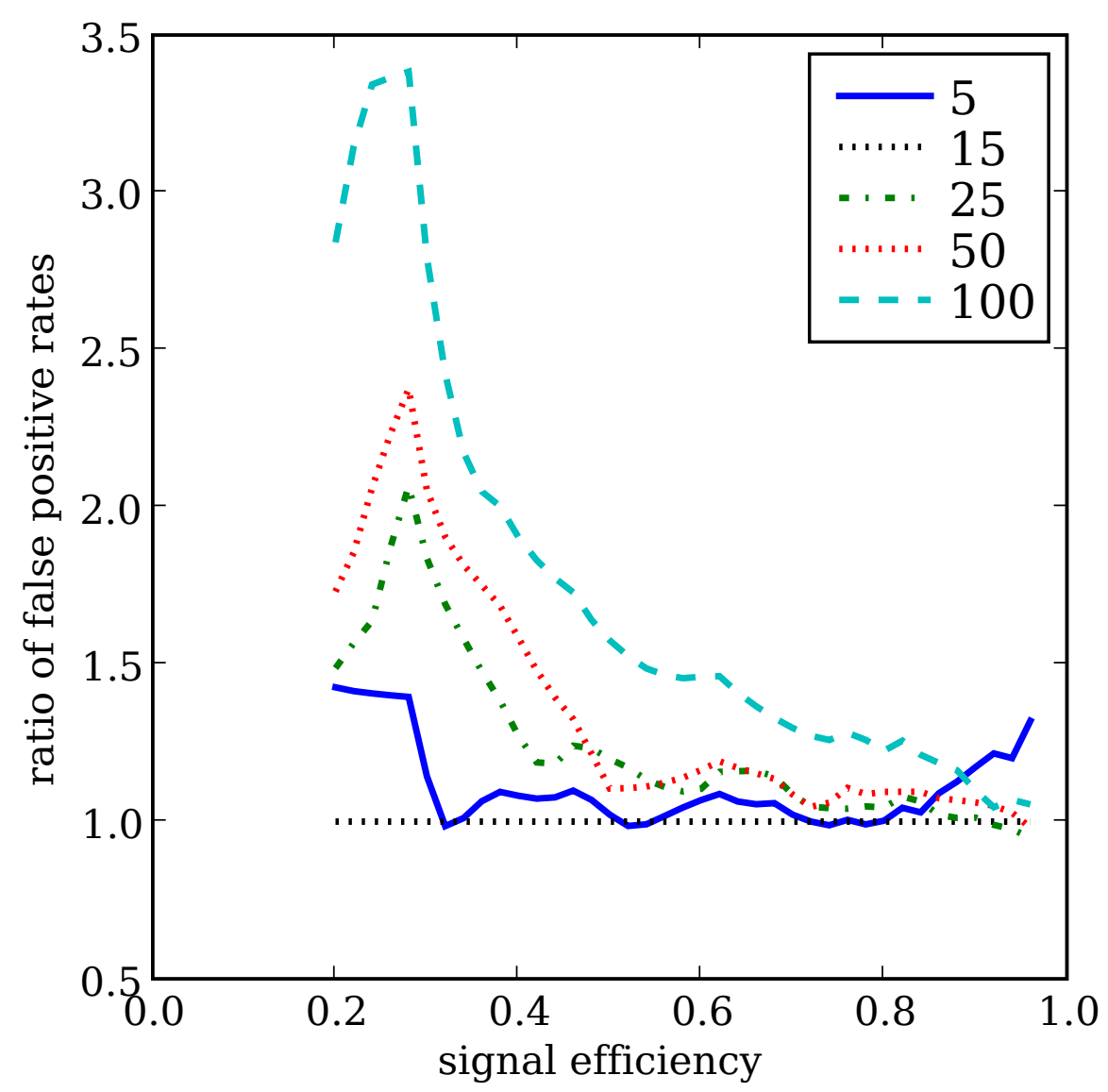

Fig. 5. - A comparison of the performance of 200 boosted trees with varying leaf sizes. 10,000 training events were used; the plot shows the comparison of leaves with a minimum of $N=5,25,50,100$ events in comparison to the performance of the $N=15$ case. 


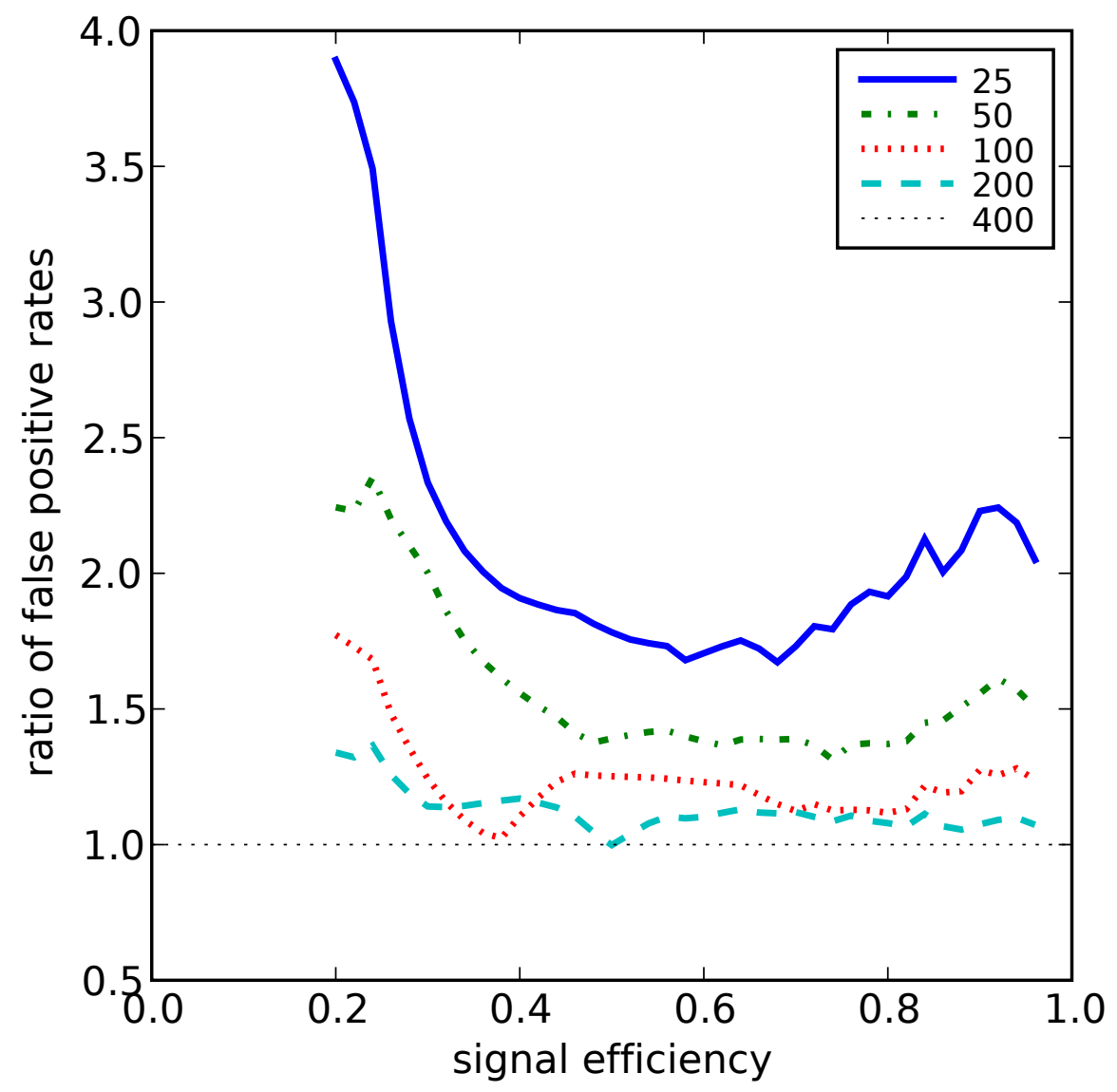

Fig. 6. - A comparison of the performance of $N_{\text {tree }}=25,50,100,200$ boosted trees with a minimum of 50 events per leaf (out of 10,000 training events) in comparison to the $N_{\text {tree }}=400$ case. 